Editorial

\title{
Miocardiopatia Adrenérgica: o Estresse Pode Causar uma Cardiopatia Aguda?
}

\author{
Adrenergic Cardiomyopathy: Can Stress Cause Acute Heart Disease?
}

\author{
Cláudio Tinoco Mesquita, Antonio Claudio Lucas da Nóbrega \\ Rio de Janeiro, RJ
}

"Todo mundo é capaz de dominar uma dor, exceto quem a sente."

\section{William Shakespeare}

A percepção de que o estresse pode estar associado a problemas cardíacos é muito antiga e não por coincidência, o coração é considerado pelo público geral, como a sede das emoções. Se por um lado, a grande aceleração da ciência ao longo do século 20 marcou uma separação das especialidades na abordagem diagnóstica e terapêutica dos pacientes ${ }^{1}$, por outro lado, tem gerado uma plêiade de evidências apontando para uma associação causal independente entre fatores como depressão, isolamento social e má qualidade de suporte social com o desenvolvimento e o prognóstico de doença arterial coronária ${ }^{2}$. 0 aumento do risco ocasionado por estes fatores psicossociais é de magnitude similar ao observado com os fatores de risco convencionais como tabagismo, dislipidemia e hipertensão, demonstrando a importância da melhor compreensão desta associação. Outro aspecto bem estudado é o impacto das emoções negativas desencadeando eventos coronarianos agudos em pessoas com doença aterosclerótica estabelecida ou com alta probabilidade de doença ${ }^{3}$.

O estresse parece estar associado a um novo tipo de cardiopatia, que vem sendo chamada no Brasil de "síndrome do coração partido". Inicialmente descrita em orientais uma nova síndrome cardíaca, caracterizada por uma disfunção ventricular esquerda transitória, tipicamente com aspecto de armadilha de pegar polvo (do japonês, Takotsubo) à ventriculografia contrastada, foi relatada em associação a situações de estresse físico ou emocional ${ }^{4,5}$. Estudos mais recentes tem contrariado uma sugestão inicial de uma predileção geográfica ou racial com a Ásia e casos em todos os continentes têm sido descritos ${ }^{6}$, sendo a maior série de casos fora do Japão publicada por pesquisadores norte americanos ${ }^{7}$. 0 caso relatado neste número dos Arquivos Brasileiros de Cardiologia é o primeiro no Brasil com esta nomenclatura e tem como um dos maiores objetivos aumentar o reconhecimento desta síndrome em nosso meio, visto que esta forma de disfunção ventricular esquerda transitória desencadeada por estresse emocional ou físico é relevante para todos os médicos, particularmente cardiologistas e emergencistas.

As características clínicas da Miocardiopatia de Takotsubo são: início súbito de dor anginosa, alterações eletrocardiográficas e eleva-

Hospital Pró-Cardíaco e Universidade Federal Fluminense Endereço para correspondência: Cláudio Tinoco Mesquita - Hospital Pró-Cardíaco - Rua Dona Mariana 219, Cep 22280-020 - Rio de Janeiro, RJ - E-mail: ctinoco@openlink.com.br ção discreta de marcadores de necrose miocárdica, compatível com infarto com supradesnível do segmento ST. Tipicamente a doença afeta mulheres idosas, com poucos fatores de risco cardiovasculares tradicionais. Os angiogramas sistematicamente evidenciam coronárias livres de obstruções significantes e um aspecto de acinesia apical característico ${ }^{8}$. A disfunção sistólica pode ser marcante, com até $40 \%$ dos pacientes requerendo estabilização hemodinâmica com vasopressores ou contrapulsação aórtica, porém a normalização da função ventricular é a regra e freqüentemente é extremamente rápida (entre 7 e 30 dias) ${ }^{7}$. Os pontos centrais e chaves na compreensão da síndrome são a predominância feminina (100\% dos casos em mulheres em algumas séries ${ }^{7,9}$ ) e a precedência imediata de um episódio de estresse físico ou psicológico intenso antes do desenvolvimento dos sintomas clínicos. São freqüentes os relatos de falecimento inesperado de um parente próximo ou amigo, abuso doméstico, diagnósticos médicos catastróficos, perdas financeiras vultuosas e situações de extrema angústia. 0 agravamento de condições médicas pré-existentes parece estar associado a um número substancial de casos, sendo associadas à síndrome, exacerbação de asma brônquica, acidente cerebrovascular, crises convulsivas, entubação orotraqueal e dor em pós-operatório ${ }^{9}$. 0 caso relatado neste número dos Arquivos está associado à insuficiência respiratória aguda em um paciente com miastenia gravis, o que está de acordo com as descrições prévias de fatores desencadeantes. O paciente descrito fazia uso regular de uma única medicação (brometo de piridostigmina) que já foi descrita como capaz de inibir, e não de exacerbar, as respostas hemodinâmicas ${ }^{10}$ e ventriculares ${ }^{11}$ ao estresse em indivíduos sem doença cardiovascular.

A base fisiopatológica da miocardiopatia de Takotsubo ainda não está clara, mas o atordoamento miocárdico parece ser o mecanismo da disfunção miocárdica. 0 atordoamento miocárdico é definido como uma disfunção ventricular pós-isquêmica prolongada como conseqüência de um período curto de isquemia. Postulase que a isquemia miocárdica seja a base das manifestações clínicas pela presença de angina e alterações eletrocardiográficas sugestivas. Este fato levou muitos autores a atribuir a um espasmo coronariano multifocal difuso ou multivascular o mecanismo da doença ${ }^{4,9}$. É extremamente improvável que o espasmo de uma única artéria, como a descendente anterior, seja a causa da síndrome, pois um recente estudo com ressonância magnética identificou alterações contráteis no território de mais de uma artéria coronariana em $95 \%$ dos casos $^{7}$. Entretanto, pesquisadores da Espanha demonstraram que todos os pacientes de uma série de 11 casos da síndrome apresentavam uma artéria descendente anterior que 
alcançava o ápice do ventrículo esquerdo e irrigava uma porção substancial da parede inferior ${ }^{12}$. Adicionalmente o mesmo grupo de autores, em outra publicação ${ }^{9}$, encontrou a presença de ruptura de placas ateroscleróticas insipientes na artéria descendente anterior através do ultra-som intracoronariano, postulando a ocorrência de uma síndrome coronariana aguda com reperfusão rápida como a causa de base da síndrome. 0 espasmo microvascular é reconhecido como causa de isquemia miocárdica ${ }^{13}$ e tem sido proposto por alguns autores como um mecanismo do atordoamento encontrado na miocardiopatia de Takotsubo ${ }^{9}$.

Níveis elevados de catecolaminas têm sido encontrados em pacientes com a Miocardiopatia de Takotsubo, sugerindo que uma estimulação simpática exagerada em resposta ao estresse seja a base da síndrome ${ }^{14}$. A hiperativação simpática em reposta ao estresse emocional aumenta os níveis circulantes de epinefrina e norepinefrina e, além de aumentar a freqüência cardíaca e o inotropismo, pode acarretar espasmo coronariano pela interação com receptores alfa-adrenérgicos ${ }^{15}$. Na realidade, a reatividade cardiovascular ao estresse mental, ou sensorial, pode ser quantificada através de testes padronizados que envolvem a exposição a uma situação controlada de conflito auditivo e visual, como o teste aritmético e o teste de cores ${ }^{16}$. A resposta hemodinâmica exacerbada ao teste de estresse sensorial tem poder prognóstico para o desenvolvimento de hipertensão arterial em indivíduos normotensos ${ }^{17}$ e para a ocorrência de eventos adversos em portadores de doenças cardiovasculares ${ }^{18}$.

Portanto, é chegada a hora do cardiologista voltar a considerar na sua prática cotidiana que processos emocionais podem participar concretamente dos complexos mecanismos envolvidos na fisiopatologia das doenças cardiovasculares.

\section{Referências}

1. Corrêa JB. Medicina psicossomática. Arq Bras Cardiol 1948; 1: 159-63.

2. Bunker SJ, Colquhoun DM, Esler MD et al. "Stress" and coronary heart disease: psychosocial risk factors. Med J Aust 2003; 17; 178: 272-6.

3. Soufer R. Neurocardiac interaction during stress-induced myocardial ischemia. Circulation 2004; 110: 1710-13.

4. Dote K, Sato H, Tateishi $\mathrm{H}$ et al. Myocardial stunning due to simultaneous multivessel coronary spasm: a review of 5 cases. J Cardiol 1991; 21:203-14.

5. Kurisu S, Sato H, Kawagoe T et al. Tako-Tsubo-like left ventricular dysfunction with ST-segment elevation: A novel cardiac syndrome mimicking acute myocardial infarction. Am Heart J 2002; 1: 43-55.

6. Gaspar J, Gomez Cruz RA. Tako-Tsubo syndrome (transient antero-apical dyskinesia): first case reported in Latin America and review of the literature. Arch Cardiol Mex 2004; 74: 205-14.

7. Sharkey S, Lesser J, Zenovich A et al. Acute and reversible cardiomyopathy provoked by stress in womem from the United States. Circulation 2005; 111: 472-9.

8. Tsuchihashi K, Ueshima K, Uchida Tet al. Transient left ventricular apical ballooning without coronary artery stenosis: a novel heart syndrome mimicking acute myocardial infarction. Angina Pectoris-Myocardial Infarction Investigations in Japan. J Am Coll Cardiol 2001; 38: 11-8.

9. Ibanez B, Navarro F, Cordoba M, M-Alberca P, Farre J. Tako-Tsubo transient left ventricular apical ballooning: is intravascular the key to resolve the enigma? Heart 2005; 91: 102-4.

10. Nóbrega AC, Carvalho AC, Santos KB, Soares PP. Cholinergic stimulation with pyridostigmine blunts the cardiac responses to mental stress. Clin Auton Res 1999; 9: 11-6.

11. Sant'anna ID, de Sousa EB, de Moraes AV, Loures DL, Mesquita ET, da Nóbrega AC. Cardiac function during mental stress: cholinergic modulation with pyridostigmine in healthy subjects. Clin Sci (Lond) 2003; 105: 161-5.

12. Ibanez B, Navarro F, Farré J et al. Tako-Tsubo transient left ventricular apical ballooning is associated with a left anterior descending coronary artery with a long course along the apical diaphragmatic surface of the left ventricle. Rev Esp Cardiol 2002004; 57: 209-16.

13. Mohri M, Koyanagi M, Egashira K et al. Angina pectoris caused by coronary microvascular spasm. Lancet 1998; 351: 1165-9.

14. Wittstein I, Thiemann D, Lima J et al. Neurohumoral Features of Myocardial Stunning Due to Sudden Emotional Stress. N Engl J Med 2005; 352:539-548.

15. Becjer L, Pepine C, Bonsall R et al. Left ventricular, peripheral vascular, and neurohumoral reponses to mental stress in normal middle-aged men and women. Circulation 1996; 94: 2768-77.

16. Loures DL, Sant Anna I, Baldotto CS, Sousa EB, Nobrega AC. Mental stress and cardiovascular system. Arq Bras Cardiol 2002; 78: 525-30.

17. Jesse C. Stewart, Christopher R. France. Cardiovascular recovery from stress predicts longitudinal changes in blood pressure. Biol Psychol 2001; 58: 105-120

18. Sheps DS, McMahon RP, Becker L et al. Mental stress-induced ischemia and all-cause mortality in patients with coronary artery disease results from the Psychophysiological Investigations of Myocardial Ischemia Study. Circulation 2002; 105: 1780-4. 